\title{
Cathepsin D Deficiency Induces Persistent Neurodegeneration in the Absence of Bax-Dependent Apoptosis
}

\author{
John J. Shacka, ${ }^{1}$ Barbara J. Klocke, ${ }^{1}$ Chainllie Young, ${ }^{2}$ Masahiro Shibata, ${ }^{3}$ John W. Olney, ${ }^{2}$ Yasuo Uchiyama, ${ }^{3}$ \\ Paul Saftig, ${ }^{4}$ and Kevin A. Roth ${ }^{1}$ \\ ${ }^{1}$ Department of Pathology, Neuropathology Division, University of Alabama at Birmingham, Birmingham, Alabama 35294, ${ }^{2}$ Department of Psychiatry, \\ Washington University School of Medicine, St. Louis, Missouri 63110, ${ }^{3}$ Department of Cell Biology and Neurosciences, Osaka University Graduate School of \\ Medicine, Suita, Osaka 565-0871, Japan, and ${ }^{4}$ Department of Biochemistry, University Kiel, D-24098 Kiel, Germany
}

Neuronal ceroid lipofuscinosces/Batten disease (NCL) is a devastating group of neurodegenerative diseases caused by genetic disruptions in lysosomal function. Cathepsin $\mathrm{D}(\mathrm{CD})$ is a major lysosomal protease, and mutations in $\mathrm{CD}$ that render it enzymatically defective have been reported recently in subsets of NCL patients. The targeted deletion of CD in mice results in extensive neuropathology, including biochemical and morphological evidence of apoptosis and autophagic stress (aberrant autophagosome accumulation), effects that are similar to those observed in NCL. To determine the contribution of Bax-dependent apoptosis in this mouse model of NCL, combined Baxand CD-deficient mice were generated. Morphological analysis of CD-deficient mouse brains indicated large numbers of pyknotic neurons and neurons with marked cytoplasmic swellings containing undigested lipofuscin. Cell death and apoptosis were evidenced by increases in terminal deoxynucleotidyl transferase-mediated biotinylated UTP nick end labeling (TUNEL) reactivity and activation of caspase-3, respectively. DeOlmos silver-positive neurons were abundant in CD-deficient brain and correlated with neuron loss, as indicated by significant decreases in NeuN (neuronal nuclear antigen)-positive neurons. Lysosome dysfunction and autophagic stress were apparent in CD-deficient brain as indicated by the accumulation of autofluorescent storage material and by increased levels of LC3-II (light chain 3-II, a selective autophagosome marker), respectively. Bax deletion significantly inhibited caspase-3 activation and hippocampal TUNEL reactivity but did not prevent the majority of CD deficiency-induced neuropathology, including the persistence of pyknotic neurons, elevated cortical TUNEL reactivity, lysosome dysfunction and autophagic stress, neurodegeneration, and neuron loss. Together, these results suggest that $\mathrm{CD}$ deficiency-induced neuropathology does not require Bax-dependent apoptosis and highlights the importance of caspase-independent neuron death and neurodegeneration resulting from the genetic disruption of lysosome function.

Key words: apoptosis; autophagic stress; autophagy; cathepsin D; Bax; neurodegeneration

\section{Introduction}

Cathepsin D (CD) hydrolyzes select peptide bonds of target proteins with high specificity and is the predominant lysosomal aspartic acid protease (Saftig et al., 1995). CD is variably expressed in all tissues and is abundantly expressed in the brain (Whitaker and Rhodes, 1983; Reid et al., 1986). Mice deficient in CD die by postnatal day $26(\mathrm{P} 26) \pm 1$ from failure to thrive, triggered by intestinal necrosis, thromboembolia, and lymphopenia (Saftig et al., 1995). The genetic disruption of lysosome function by CD deficiency induces a lysosomal storage disorder characterized in the brain by the progressive accumulation of undigested lipopigment and the accumulation of proteins including subunit $\mathrm{c}$ of

Received Aug. 18, 2006; revised Jan. 17, 2007; accepted Jan. 20, 2007.

This work was supported by National Institutes of Health (NIH) Grants NS35107 and NS41962 (K.A.R.) and by an award from the Batten Disease Support and Research Association (J.J.S.). We thank Cecelia B. Latham and University of Alabama at Birmingham Neuroscience Core Facilities (NIH Grants NS47466 and NS57098) for expert technical assistance.

Correspondence should be addressed to Dr. Kevin A. Roth, Department of Pathology, Neuropathology Division, University of Alabama at Birmingham, SC 9611530 3rd Avenue South, Birmingham, AL 35294-0017. E-mail: karoth@uab.edu.

DOI:10.1523/JNEUROSCI.5577-06.2007

Copyright $\odot 2007$ Society for Neuroscience $\quad$ 0270-6474/07/272081-10\$15.00/0 mitochondrial $\mathrm{F}_{1} \mathrm{~F}_{0} \mathrm{ATPase}$ (Koike et al., 2000), features similar to that found in CLN-2, a late infantile form of neuronal ceroid lipofuscinosces/Batten disease (NCL) (Haltia, 2003; Goebel and Wisniewski, 2004).

NCL is a group of fatal, autosomal recessive pediatric neurodegenerative diseases that lack effective treatment (Haltia, 2003). Interestingly, levels of the protein tripeptidyl peptidase I increase in CD-deficient mouse brain but decrease in human NCL-2, suggesting that $\mathrm{CD}$ deficiency defines a novel subtype of NCL (Koike et al., 2000, 2005; Shacka and Roth, 2005). Mutations in CD with dramatically reduced enzymatic activity and resultant neurodegeneration have been reported in sheep (congenital ovine NCL) and American bulldogs (Tyynela et al., 2000; Awano et al., 2006). CD mutations have been reported recently in humans, and case studies suggest the potential importance of CD activity levels on age of onset of NCL-like symptoms (Siintola et al., 2006; Steinfeld et al., 2006). Significant decreases in CD activity have been documented in a teenaged patient (Steinfeld et al., 2006), whereas a complete absence of CD activity was associated with congenital NCL and early neonatal mortality (Siintola et al., 2006).

CD-deficient neurons exhibit increased markers that indicate activation of the intrinsic apoptotic pathway that is critically me- 
diated by Bax, a pro-apoptotic member of the Bcl-2 family. On receipt of a death signal, Bax translocates to mitochondria and induces release of cytochrome $c$, which forms a critical component of the apoptosome and leads ultimately to activation of effector caspases and cell death (Jurgensmeier et al., 1998; Shacka and Roth, 2006). The importance of Bax in apoptotic cell death is underscored by in vitro and in vivo studies of its targeted deletion, which indicate attenuation of neuron apoptosis and increased survival, both during nervous system development and in models of neuron death (Deckwerth et al., 1996; Shindler et al., 1997, 1998; White et al., 1998).

The term "autophagic stress" is used to define the aberrant accumulation of autophagosomes in cells and may be related to specific compromises in macronutrient degradation (Chu, 2006). Autophagic stress in CD-deficient brain has been shown previously to precede markers of apoptosis, suggesting that $\mathrm{CD}$ deficiency-induced lysosome dysfunction may contribute to the induction of apoptosis (Koike et al., 2000, 2005; Nakanishi et al., 2001; Walls et al., 2007). However, because the relative importance of apoptosis to CD deficiency-induced neuropathology is poorly defined, combined Bax- and CD-deficient mice were generated, and markers of cell death, apoptosis, neurodegeneration, and autophagic stress were assessed. Although Bax deficiency clearly reduced apoptosis in CD-deficient brain, it did not attenuate non-apoptotic death, neurodegeneration, or autophagic stress.

\section{Materials and Methods}

Animals. C57BL/6J mice were used in all experiments. Mice were cared for in accordance with the guidelines of the National Institutes of Health Guide for the Care and Use of Laboratory Animals. All animal protocols were approved by the Institutional Animal Care and Use Committee of the University of Alabama at Birmingham. The generation and characterization of mice deficient in bax and cathepsin $d$ has been described previously (Knudson et al., 1995; Saftig et al., 1995; Shindler et al., 1997). Genotyping of bax was determined by PCR analysis of tail DNA extracts as described previously (Shindler et al., 1997). Genotyping of cathepsind was performed using primers specific for the wild-type (WT) allele ( $5^{\prime}$ AGACTAACAGGCCTGTTCCC and 3'-TCAGCTGTAGTTGCTCACATG) and primers specific for the knock-out allele (5'-CTCGTCCTGCAGTTCATTCA and 3'-CCCCTCAGCTGTAGTTGCTC). Mice that were determined to be heterozygous and homozygous dominant for Bax and CD were pooled together for the WT control group, except where indicated. Mice were weighed daily beginning on P8 and killed on P26 or earlier if they appeared in distress. For DeOlmos silver stain histochemistry, animals were perfused transcardially with saline, followed by $4 \%$ paraformaldehyde, $\mathrm{pH} 7.4$, in $0.1 \mathrm{M}$ Tris. Perfused brains were subsequently postfixed in $4 \%$ paraformaldehyde, $0.9 \%$ sodium chloride, and $0.1 \%$ sodium cacodylate for at least $2 \mathrm{~d}$ before sectioning. For all other immunohistochemistry and Western blot analysis, brains obtained from nonperfused animals were cut sagitally along the midline; one-half of each brain was frozen immediately for Western blot analysis, whereas the other half was submerged in Bouin's fixative overnight at $4^{\circ} \mathrm{C}$ for immunohistochemical analysis.

Hematoxylin and eosin stain. Five-micrometer-thick sagittal sections from paraffin-embedded brains were deparaffinized with Citrisolv (Fisher Scientific, Pittsburgh, PA) and isopropanol, followed by incubation with Harris's modified hematoxylin with acetic acid (Fisher Scientific) for $90 \mathrm{~s}$, and rinsed with distilled $\mathrm{H}_{2} \mathrm{O}$. Hematoxylin-stained slides were next decolorized by a $5 \mathrm{~s}$ dip in acid-ethanol $(1 \%$ concentrated $\mathrm{HCl}$ in $70 \%$ ethanol) and incubated with bluing reagent (1\% ammonium hydroxide in distilled $\mathrm{H}_{2} \mathrm{O}$ ) for 15-20 s, followed by distilled $\mathrm{H}_{2} \mathrm{O}$ wash. Slides were next incubated with Eosin Y (Surgipath Medical Industries, Richmond, IL) for $75 \mathrm{~s}$, followed by incubation with ethanol (95 and $100 \%$ ) and xylene, and mounted with Cytoseal 60.

Microscopy and image capture. All images from tissue sections were examined with a Zeiss (Thornwood, NY) Axioskop microscope equipped with epifluorescence, and images were captured using Zeiss Axiovision software. All images were exported subsequently to Adobe Photoshop (Adobe Systems, Mountain View, CA) for presentation.

Autofluorescence. Five-micrometer-thick sagittal sections from paraffin-embedded brains were deparaffinized as above and coverslipped with PBS-glycerol (1:1). Autofluorescence was visualized using a $470 \mathrm{~nm}$ short-pass excitation filter and a $520 \mathrm{~nm}$ long-pass emission filter. Images with an area of $0.4 \mu \mathrm{m}^{2}$ were captured using equal exposure times and equal, nonsaturating threshold levels as determined by Zeiss Axiovision software. Pixel intensity (designated as autofluorescence units) for each image was counted after export of images to Adobe Photoshop. Images were captured from three contiguous fields of the CA1-CA2 hippocampal region, the cortex (immediately dorsal to the CA1 hippocampal region), and thalamus. For each brain region, autofluorescence units from the three contiguous fields were averaged together. Autofluorescence units were also calculated from one $0.4 \mu \mathrm{m}^{2}$ image captured from within the subiculum, an area that relays synaptic information between the CA1 hippocampal region and the cortex and was defined anatomically in sagittal sections as the area immediately posterior to the CA1 region of the hippocampus and ventral to the cortex and corpus callosum.

Chromogenic in situ detection of cleaved caspase-3, neuronal nuclear antigen, and light chain 3. Five-micrometer-thick sagittal sections from paraffin-embedded brains were deparaffinized as above, subjected to antigen retrieval in citrate buffer with heat, and incubated for $5 \mathrm{~min}$ with $3 \% \mathrm{H}_{2} \mathrm{O}_{2}$ in $\mathrm{PBS}$ to reduce endogenous peroxidase activity. Sections were incubated in PBS blocking buffer (PBSBB; $1 \%$ BSA, $0.2 \%$ evaporated milk, and $0.3 \%$ Triton X-100 in PBS) for $30 \mathrm{~min}$, followed by overnight $\left(4^{\circ} \mathrm{C}\right)$ incubation in $\mathrm{PBSBB}$ with mouse anti-neuronal nuclear antigen (NeuN; Chemicon, Temecula, CA), rabbit anti-cleaved caspase-3 (Cell Signaling, Beverly, MA), or rabbit anti-light chain 3 (LC3) (Lu et al., 2005) primary antibodies. After PBS washes, slides were incubated with HRP-conjugated secondary antibodies (donkey anti-rabbit or antimouse IgG, $1 \mathrm{~h}$ at room temperature; Jackson ImmunoResearch , West Grove, PA), followed by PBS wash. Antibody localization was detected by Tyramide Signal Amplification (PerkinElmer Life Sciences, Norwalk, CT) using biotin-conjugated tyramide (30 min at room temperature, followed by PBS wash), followed by the addition of streptavidin-HRP ( $1 \mathrm{~h}$ at room temperature, followed by PBS wash) and DAB chromogen (2-5 min at room temperature, followed by distilled $\mathrm{H}_{2} \mathrm{O}$ wash; Pierce, Rockford, IL). Slides were counterstained with hematoxylin, decolorized with acid-ethanol, incubated with bluing reagent as above, dehydrated in increasing percentages of ethanol, cleared in xylene, and coverslipped with Cytoseal 60.

Chromogenic in situ detection of DNA fragmentation. The ApopTag Plus Peroxidase In Situ Apoptosis Detection kit (Chemicon) was performed according to the manufacturer's instructions with minor modifications as described previously (Tornusciolo et al., 1995). Fivemicrometer-thick paraffin-embedded sagittal brain sections from five different mice of each genotype were deparaffinized as above, permeabilized for 10 min with $0.5 \%$ Triton X-100, oxidized for 5 min with $0.3 \%$ $\mathrm{H}_{2} \mathrm{O}_{2}$ in PBS to reduce endogenous peroxidase activity, and subsequently washed with PBS. Slides were next prehybridized in equilibration buffer for $5 \mathrm{~min}$, followed by hybridization with terminal deoxynucleotidyl transferase (TDT) enzyme for $2 \mathrm{~h}$ at $37^{\circ} \mathrm{C}$. The hybridization reaction was terminated by incubation with stop buffer and PBS wash. HRPconjugated anti-digoxigenin was added next (overnight at $4^{\circ} \mathrm{C}$ ), followed by PBS wash and chromagenic detection (15-20 min) using DAB metal (Pierce). Slides were counterstained with hematoxylin, decolorized with acid-ethanol, incubated with bluing reagent as above, dehydrated in increasing percentages of ethanol, cleared in xylene, and coverslipped with Cytoseal 60. Images with an area of $0.4 \mu \mathrm{m}^{2}$ were captured from both the cortex (four contiguous fields immediately dorsal to the hippocampus) and five to seven contiguous fields encompassing the length of the CA1-CA2 region of the hippocampus. The numbers of TDTmediated biotinylated UTP nick end labeling (TUNEL)-reactive cells were counted in separate fields using Image Pro Plus-4 software, subsequently totaled for each brain region (ranging from 400 to 700 cells for the CA1-CA2 region of the hippocampus and from 500 to 700 cells for 
the cortex), and expressed as a percentage of the total number of nuclei counted in these corresponding fields.

$\mathrm{DeOlmos}$ silver stain. Silver staining was performed as a modification of a previous protocol (DeOlmos and Ingram, 1971). Brains were perfused ( $4 \%$ paraformaldehyde, $\mathrm{pH} 7.4$, in $0.1 \mathrm{~m}$ Tris) and postfixed in $4 \%$ paraformaldehyde, $0.9 \%$ sodium chloride, and $0.1 \%$ sodium cacodylate at least $2 \mathrm{~d}$ before sectioning. Brains were then cut sagitally using a vibrating microslicer (70 $\mu \mathrm{m}$ thick). Free-floating sections were rinsed in deionized water and, after overnight incubation in cupric silver solution, were washed in acetone and stained with silver diammine solution. Sections were reduced in citric acid solution, rinsed with deionized water, bleached with $0.3 \% \mathrm{~K}_{3} \mathrm{Fe}(\mathrm{CN})_{6}$, and stabilized with $0.1 \% \mathrm{Na}_{2} \mathrm{~S}_{2} \mathrm{O}_{3}$. Sections were mounted on gel-coated slides, dried overnight, dehydrated in increasing concentrations of ethanol $(30-100 \%)$, cleared with xylene, and mounted with Cytoseal 60 . The numbers of silver-positive neurons were counted using Image Pro Plus- 4 software and averaged together from three different brains of animals representing each genotype. Brain regions assessed for neurodegeneration included the subiculum (one 0.4 $\mu \mathrm{m}^{2}$ field defined anatomically as immediately posterior to the CA1 hippocampal region and ventral to the cortex and corpus callosum), the CA1 region of the hippocampus (five to seven contiguous $0.4 \mu \mathrm{m}^{2}$ fields representing $1 \mathrm{~mm}$ in length of the CA1 region), and the cortex (four contiguous $0.4 \mu \mathrm{m}^{2}$ fields located immediately dorsal to the hippocampus).

Quantitation of neuron loss. The numbers of NeuN-immunoreactive cells (NeuN with the immunohistochemical protocol described above) were counted using Image Pro Plus- 4 software and averaged together from brains of at least seven different mice representing each genotype. Brain regions assessed for neuron loss included the subiculum (one 0.4 $\mu \mathrm{m}^{2}$ field defined anatomically as immediately posterior to the CA1 hippocampal region and ventral to the cortex and corpus callosum), the CA1 region of the hippocampus (five to seven contiguous $0.4 \mu \mathrm{m}^{2}$ fields representing $1 \mathrm{~mm}$ in length of the CA1 region), and the cortex (four contiguous $0.4 \mu \mathrm{m}^{2}$ fields located immediately dorsal to the hippocampal CA1 region). The numbers of NeuN-immunoreactive cells from different fields of the same brain region of the same mouse were totaled together, and a group average was calculated.

Western blot. Hemibrains were homogenized in lysis buffer containing 25 mm HEPES, 5 mм EDTA, 5 mm $\mathrm{MgCl}_{2}$, 1\% SDS, 1\% Triton X-100, 1 mM PMSF, $1 \%$ protease inhibitor mixture, and $1 \%$ phosphatase inhibitor mixture (Sigma). Equal amounts of protein were resolved via SDS-PAGE and transferred to polyvinylidene difluoride. Blots were blocked for $1 \mathrm{~h}$ at room temperature (5\% milk), followed by an overnight incubation with antibodies for cleaved caspase- 3 or LC3 (see above), or for rabbit anti- $\beta$ tubulin (Santa Cruz Biotechnology, Santa Cruz, CA), which served as a loading control. Blots were washed with $1 \times$ TBS containing $0.1 \%$ Tween 20 , incubated with a secondary antibody (goat anti-rabbit IgG, $1 \mathrm{~h}$ at room temperature), and washed. Signal was detected using Supersignal chemiluminescence (Pierce). Blots were scanned for densitometric analysis using Bio-Rad (Hercules, CA) Quantity One software.

Statistics. For all experiments, each genotype tested (WT, Bax deficiency, CD deficiency, and combined Bax and CD deficiency) was represented by at least three different mice from at least three different litters. Significant effects of genotype were determined via one-factor ANOVA, and age-dependent effects of genotype on pup weights were determined via two-factor ANOVA. Post hoc analysis was conducted using Bonferonni's test. A level of $p<0.05$ was considered significant.

\section{Results}

Previous investigation of CD-deficient mice indicated a failure to thrive beginning in their third postnatal week, with subsequent death by P26 \pm 1 (Saftig et al., 1995). To determine whether Bax deficiency altered this general decline in health induced by CD deficiency, pups were weighed daily from P8 to P26 (Fig. 1). WT control mice registered consistent increases in weight throughout this time period. Bax-deficient mice also consistently gained weight, although they weighed slightly less than WT control mice after P20. Although Bax-deficient mice appeared otherwise nor-

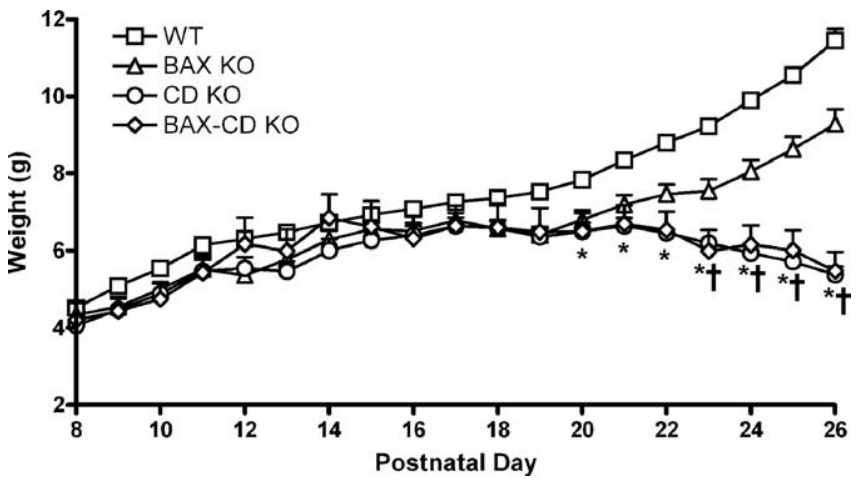

Figure 1. Bax deficiency (Bax KO) does not prevent decreased weight gain induced by $C D$ deficiency (CD K0). Mice were weighed beginning on P8 until they were killed on or around P26. ${ }^{*} p<0.05$, significantly different from WT control mice; ${ }^{\dagger} p<0.05$, significantly different from Bax-deficient mice. Data represent mean \pm SEM from at least three separate litters of mice.

mal compared with WT controls, as described below, it is still possible that endocrinological, neurological, or metabolic differences exist between these two groups of mice that could possibly result in genotype-specific effects in brain function later in life. Both CD-deficient mice and combined Bax- and CD-deficient mice weighed significantly less than WT control mice from P20 to P26 and weighed significantly less than Bax-deficient mice from P23 to P26. However, Bax deficiency did not rescue the CD deficiency-induced weight loss, which was statistically similar in both CD-deficient and combined Bax- and CD-deficient groups of mice from P20 to P26.

The effect of targeted Bax deletion on CD deficiency-induced neuropathology was assessed initially by analysis of hematoxylin and eosin-stained sections from P25-P26 mice (Figs. 2, 3). WT control and Bax-deficient control brains showed no evidence of apoptotic histological changes (Figs. $2 A, B, 3 A, B$ ). In many regions of CD-deficient brain (including the cortex, CA1-CA2 hippocampal region, thalamus, and subiculum), there were numerous pyknotic cells with classical apoptotic features including condensed, fragmented, and hyperchromatic nuclei; apoptotic bodies; and marginated chromatin (Figs. $2 C, 3 C, E$ ). The majority of cells in CD-deficient brain that exhibited apoptotic morphology also exhibited hypereosinophilic cytoplasm, although there were also cells exhibiting hypereosinophilic cytoplasm without nuclear condensation or fragmentation (Figs. $2 C, 3 C, E$ ). In addition, there were cells with swollen, lipofuscin-laden cytoplasm in CD-deficient brain that lacked apoptotic morphology (Fig. 2C). Pyknotic, hypereosinophilic cells and cells with swollen, lipofuscin-laden cytoplasm were still observed in the brains of combined Bax- and CD-deficient mice with a distribution similar to that of CD-deficient brain (Figs. 2D, 3D,F). However, much of the apoptotic morphology exhibited in CD-deficient brain (including nuclear fragmentation, apoptotic bodies, and marginated chromatin) was rare in cells of combined Bax- and CDdeficient brain. Furthermore, there was an apparent reduction in CA1-CA2 hippocampal neurons with abnormal morphology in the combined Bax- and CD-deficient brains (Fig. 3D,F).

To quantify cell death in situ, the TUNEL assay was performed, which is a sensitive marker of DNA fragmentation that occurs with both apoptosis and necrosis (Tornusciolo et al., 1995; van Lookeren et al., 1995). The numbers of TUNEL-reactive cells were counted from both the cortex (Fig. 2) and the CA1-CA2 hippocampal region (Fig. 3). WT and Bax-deficient cortex and hippocampus exhibited minimal TUNEL reactivity (Figs. 2E, 


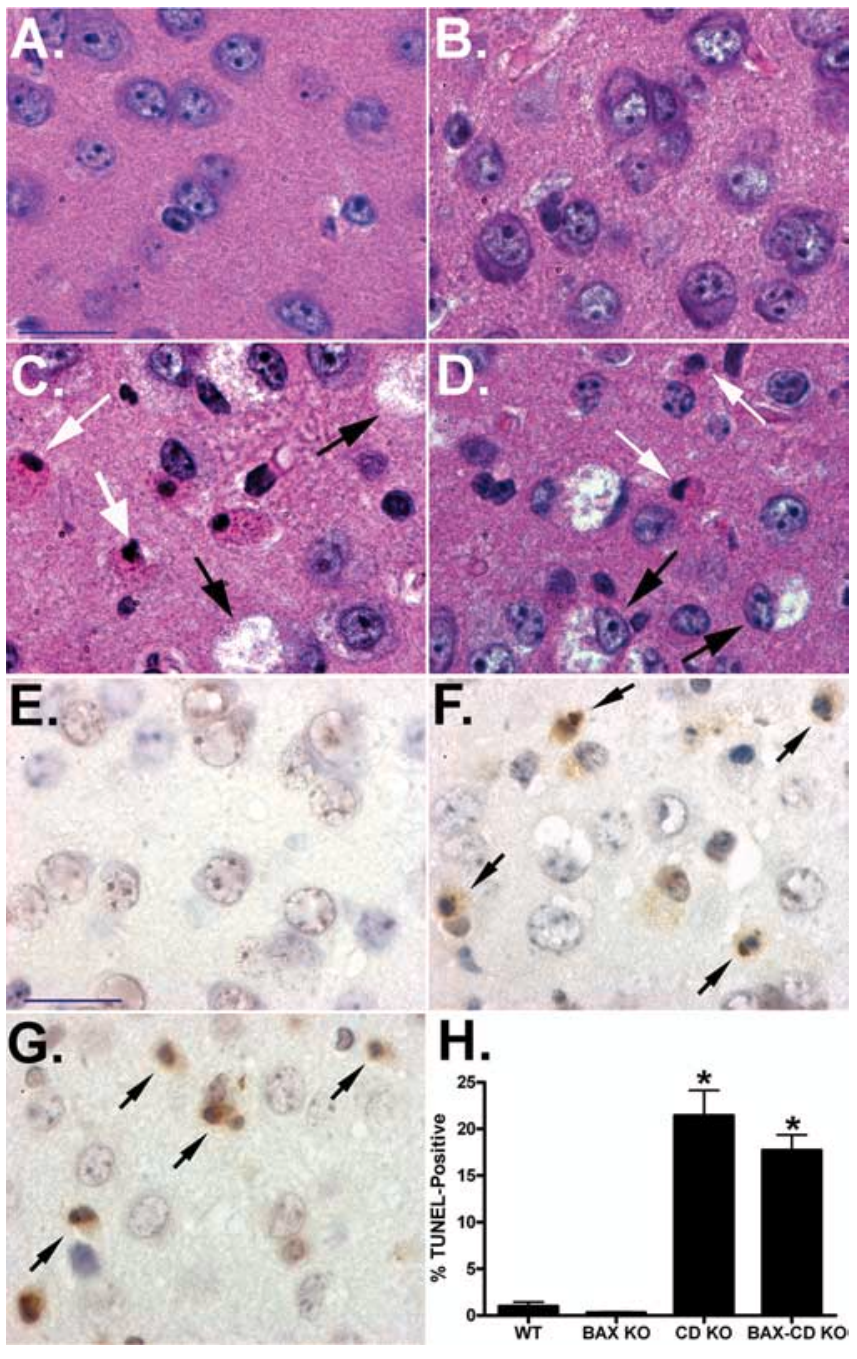

Figure 2. Targeted Bax deletion does not attenuate $C D$ deficiency-induced neuropathology and cell death, as assessed by hematoxylin and eosin stain $(\boldsymbol{A}-\boldsymbol{D})$ or TUNEL reactivity $(\boldsymbol{E}-\boldsymbol{H}) . \boldsymbol{A}$, $\boldsymbol{B}$, WT controls $(\boldsymbol{A})$ and Bax-deficient controls $(\boldsymbol{B})$ lack any gross neuropathology and exhibit an absence of TUNEL-reactive cells. C, D, $\boldsymbol{F}, \mathbf{G}$, CD-deficient cortex exhibits an abundance of cells with hypereosinophilic cytoplasm and pyknotic, hyperchromatic nuclei ( $\boldsymbol{C}$, white arrows); cells with swollen cytoplasm ( $\boldsymbol{C}$, black arrows); and TUNEL-reactive cells $(\boldsymbol{F}$, black arrows), which are still present in combined (D- and Bax-deficient cortex ( $\boldsymbol{D}$, white arrows, $\boldsymbol{G}$, black arrows). Similar findings were observed in the thalamus and subiculum (data not shown). $\boldsymbol{H}$, Quantitation of TUNEL-reactive cells is represented graphically as mean \pm SEM. ${ }^{*} p<0.05$, significantly different from WT and Bax-deficient controls. Results are representative of images obtained from at least three separate litters of mice. Scale bars, $20 \mu \mathrm{m}$. BAX K0, Bax-deficient; CD K0, CDdeficient; BAX-CD K0, combined Bax- and CD-deficient.

$3 G)$. A significant increase in the percentage of TUNEL-reactive cells was observed in both CD-deficient $(21 \pm 6 \%)$ (Fig. $2 F, H)$ and combined Bax- and CD-deficient (18 \pm 3.7\%) (Fig. 2G,H) cortex compared with WT control $(1 \pm 1 \%)$ (Fig. $2 E, H)$ and Bax-deficient control $(0.3 \pm 0.2 \%)$ (Fig. $2 H)$, whereas no difference was observed between CD-deficient and combined Bax- and CD-deficient cortex. A significant increase in the percentage of TUNEL-reactive cells was also observed in CD-deficient CA1CA2 hippocampal region $(14 \pm 3.3 \%$ ) (Fig. $3 \mathrm{H}, \mathrm{J})$ compared with WT control $(0.5 \pm 0.3 \%)$ (Fig. $3 G, J)$. In contrast to observations from the cortex, only a small percentage $(3.6 \pm 1.3 \%)$ of TUNEL-reactive cells was observed in the combined Bax- and CD-deficient CA1-CA2 hippocampal region (Fig. $3 I$, J), which was significantly less than that observed in CD-deficient hip-
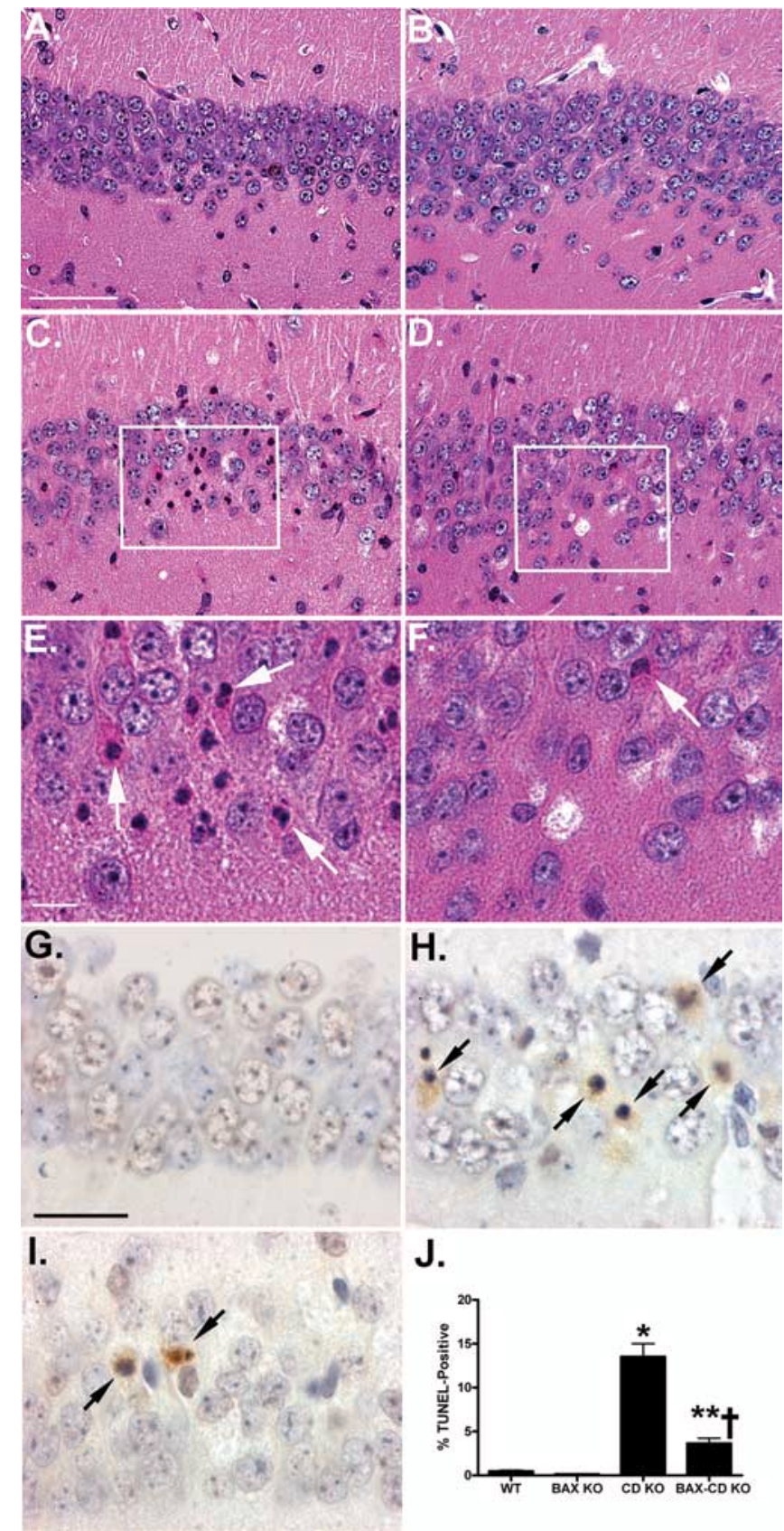

J.

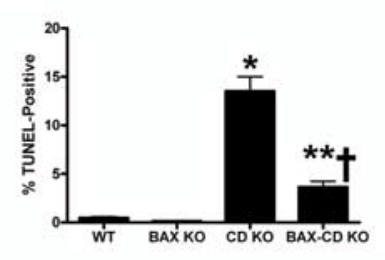

Figure 3. Targeted Bax deletion provides partial protection against $C D$ deficiency-induced neuropathology selective for the CA1-CA2 region of the hippocampus, as assessed by hematoxylin and eosin stain $(\boldsymbol{A}-\boldsymbol{F})$ or TUNEL reactivity $(\boldsymbol{G}-\boldsymbol{J})$. Boxes in $\boldsymbol{C}$ and $\boldsymbol{D}$ correspond to highermagnification images of $\boldsymbol{E}$ and $\boldsymbol{F}$, respectively. $\boldsymbol{A}, \boldsymbol{B}$, WT controls $(\boldsymbol{A})$ and Bax-deficient controls (B) lack gross neuropathology and TUNEL-reactive cells. $\mathbf{C}-\boldsymbol{E}, \boldsymbol{H}$, The CD-deficient CA1-CA2 hippocampal region $(\boldsymbol{C}, \boldsymbol{E}, \boldsymbol{H})$ exhibits abundant cells with hyperchromatic, pyknotic nuclei and hypereosinophilic cytoplasm ( $\boldsymbol{E}$, arrows) and TUNEL-reactive cells ( $\boldsymbol{H}$, arrows), which are reduced in combined $(D$ - and Bax-deficient brain (D). $J$, The percentage of TUNEL-reactive cells is represented graphically as mean \pm SEM. *Significantly different from wild type (WT); **significantly different from Bax deficient (BAX K0); ${ }^{\dagger}$ significantly different from combined Bax and CD deficient (CD KO) $(p<0.05)$. Results are representative of images obtained from at least three separate litters of mice. Scale bars: $A-D, 50 \mu \mathrm{m} ; \boldsymbol{E}, \boldsymbol{F}, 10 \mu \mathrm{m} ; \mathbf{G}-\mathbf{I}, 20 \mu \mathrm{m}$.

pocampus yet significantly greater than that of Bax-deficient controls $(0.1 \pm 0.1 \%)$. TUNEL-reactive cells were also found consistently in both CD-deficient and combined Bax- and CD-deficient thalamus and subiculum (data not shown).

To confirm that the effects of CD deficiency were not influ- 
A. Cortex

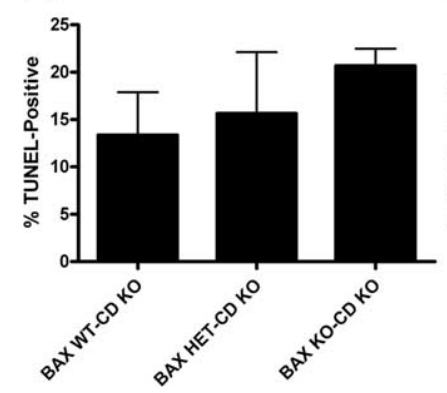

B. Hippocampal CA1-2

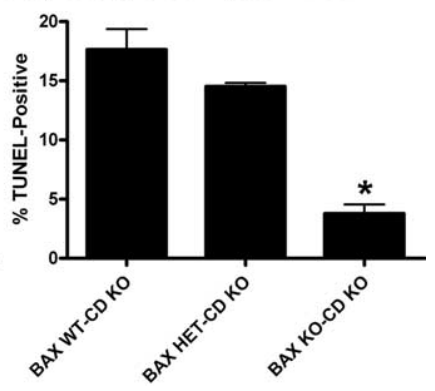

Figure 4. $\quad A, B$, The percentage of TUNEL-reactive cells in CD-deficient (CD KO) cortex $(A)$ or in the CA1-CA2 region of the hippocampus ( $\boldsymbol{B}$ ) is not affected by Bax heterozygosity (HET). The percentage of TUNEL-reactive cells is represented graphically as mean \pm SEM from four separate litters of mice. ${ }^{*} p<0.05$, significantly different from BAX WT-CD KO and BAX HET-CD KO.

enced by bax gene dosage, the percentage of TUNEL-reactive cells was assessed in brains of CD-deficient mice that were separated into three groups according to bax genotype (homozygous WT, heterozygous, and homozygous Bax deficient). There was no significant difference in the percentage of TUNEL-reactive cells in the cortex (Fig. 4A) of these three different groups of CDdeficient mice, which is similar to results observed in Figure 2. In the CA1-CA2 region of the hippocampus, the percentage of TUNEL-reactive cells was statistically similar in CD-deficient mice that had either homozygous WT or heterozygous Bax genotypes (Fig. $4 B$ ). The percentage of TUNEL-reactive cells in the CA1-CA2 hippocampal region of combined Bax- and CDdeficient mice was significantly reduced compared with CDdeficient mice that were either homozygous WT or heterozygous for bax, which is similar to results observed in Figure 3. Together, these results indicate that Bax heterozygosity did not offer any protection against CD deficiency-induced neuropathology.

The effect of genotype on the extent of apoptotic death in the brain was quantified by Western blot analysis of whole-brain lysates (Fig. $5 A, B$ ) and assessed by immunohistochemistry (Fig. $5 C$ ) for the cleaved "active" form of caspase-3. Cleaved caspase-3 was not detected in lysates prepared from WT or Bax-deficient control brains and was robust and significantly greater (migrating at $17 \mathrm{kDa}$ ) in lysates prepared from CD-deficient brain. Relative to brain extracts from CD-deficient mice with one or two copies of WT bax, cleaved caspase- 3 was reduced significantly in combined Bax- and CD-deficient brain lysates. Immunohistochemical analysis of cleaved caspase-3 confirmed the results of Western blot analysis. Immunoreactivity for cleaved caspase-3 was virtually absent in brains of WT and Bax-deficient control mice (Fig. $5 \mathrm{Ca}, \mathrm{Cb}$ ) but was robust in CD-deficient cortex (Fig. $5 C c$ ), CA1-CA2 hippocampal region, thalamus, and subiculum (data not shown). Cleaved caspase-3-immunoreactive cells were rare in brains of mice with combined Bax and CD deficiency (Fig. $5 C d$ ), indicating that Bax deficiency markedly attenuated CD deficiency-induced activation of caspase-3. In addition, a population of cells with pyknotic, hyperchromatic nuclei was observed in CD-deficient cortex (Fig. 5Cc), CA1-CA2 hippocampal region, thalamus, and subiculum (data not shown) that was not immunoreactive for cleaved caspase- 3 and may represent latestage apoptosis typified by a decrease in cleaved caspase- 3 from peak levels. However, this population of cleaved caspase-3negative cells with pyknotic, hyperchromatic nuclei may also signify a population of cells undergoing caspase-independent cell death. This possibility is supported by a morphologically similar population of pyknotic, cleaved caspase-3-negative cells in com-
A.

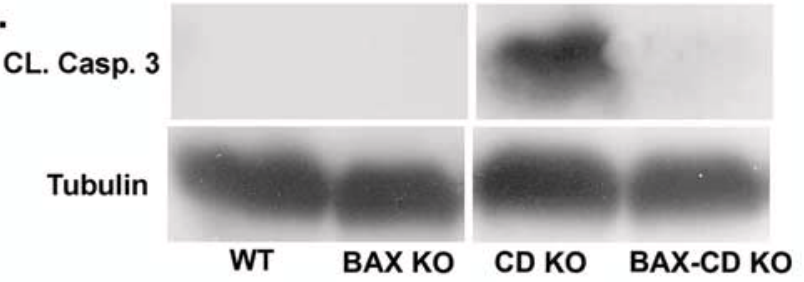

B.

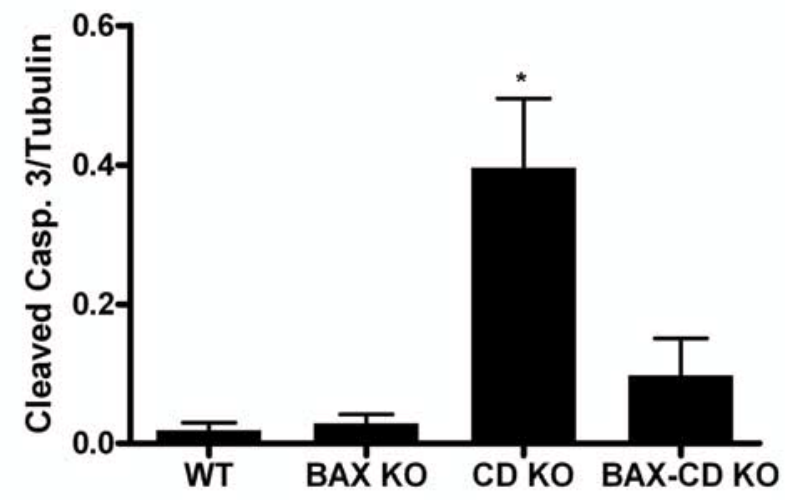

C.
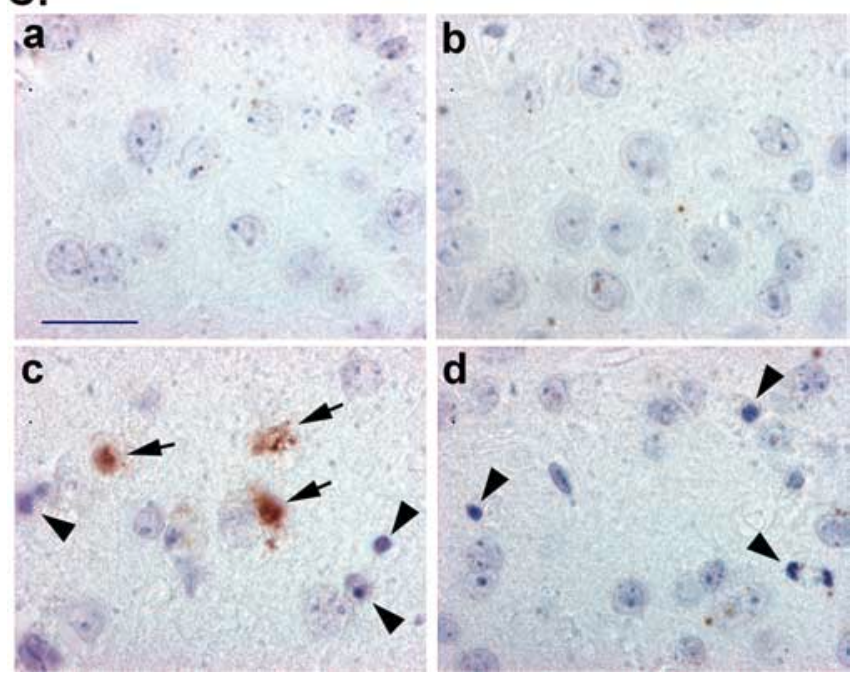

Figure 5. Bax deficiency prevents the activation of caspase-3 induced by CD deficiency. $A$, Western blot analysis of whole-brain lysates obtained from P26 mice indicates the migration of cleaved caspase-3 (CL. Casp. 3) at $\sim 17 \mathrm{kDa}$. $\beta$-Tubulin, migrating at $\sim 50 \mathrm{kDa}$, was used as a loading control. $\boldsymbol{B}$, Results of Western blot for the ratio of cleaved caspase-3 (Casp. 3)/ $\beta$-tubulin are represented graphically as mean \pm SEM. ${ }^{*} p<0.05$, significantly different from WT, Baxdeficient, and combined Bax- and CD-deficient mice. C, Immunohistochemical analysis of cleaved caspase-3 in cortex from P26 WT control (a), Bax-deficient (b), CD-deficient (c), and combined Bax-and CD-deficient (d) mice. Arrows indicate cells with positive immunoreactivity for cleaved caspase-3. Arrowheads indicate cells with pyknotic nuclei that lack cleaved caspase-3 immunoreactivity. Results are representative of measurements from three separate litters of mice. Scale bar, $20 \mu \mathrm{m}$. BAX KO, Bax-deficient; CD K0, CD-deficient; BAX-CD KO, combined Bax- and CD-deficient.

bined Bax- and CD-deficient cortex (Fig. $5 C d$ ) and in other brain regions.

Because our morphological assessments and results of TUNEL reactivity and cleaved caspase- 3 immunoreactivity revealed persistent cell death in the absence of Bax-dependent apoptosis, the relationship of cell death to neurodegeneration was assessed using the DeOlmos cupric-silver stain protocol (Fig. 6), which indicates dying or degenerating neurons via their impregnation with silver (DeOlmos and Ingram, 1971). Three brain regions were quantified: the CA1 hippocampal region (Fig. 
$6 A, B$ ), cortex (Fig. $6 C, D$ ), and subiculum (Fig. 6E,F). A virtual absence of silverpositive neurons was apparent in all WT (Fig. 6Aa,Ca,Ea) and Bax-deficient (Fig. $6 A b, C b, E b)$ brain regions. In sharp contrast, CD-deficient (Fig. 6Ac,Cc,Ec) and combined Bax- and CD-deficient (Fig. $6 A d, C d, E d)$ mice exhibited robust numbers of silver-positive neurons in these three brain regions (Fig. 6C,D). This pattern of neurodegeneration was also present in other brain regions, including but not limited to the CA2 and CA4 hippocampal regions and the thalamus (data not shown). To quantify the effect of genotype on neurodegeneration, the numbers of silver-positive neurons were counted in the CA1 region of the hippocampus (Fig. $6 B$ ), the cortex (Fig. $6 D$ ), and the subiculum (Fig. $6 F$ ). The numbers of silver-positive neurons were dramatically and significantly greater in each of these regions of CD-deficient brain compared with WT and Bax-deficient controls, an effect that was statistically similar in these regions of combined Bax- and CD-deficient brains.

To correlate the increase in cell death and neurodegeneration observed in CDdeficient and combined Bax- and CDdeficient brains with potential changes in neuron number, NeuN immunoreactivity (a nuclear marker selective for neurons) was quantified in the CA1 hippocampal region (Fig. $7 A$ ), cortex (Fig. $7 B$ ), and subiculum (Fig. 7C). The total number of NeuN-positive neurons counted in WT and Bax-deficient brain regions was statistically similar, whereas significant reductions in neuron number were observed in each brain region examined in CDdeficient mice as well as in combined Baxand CD-deficient mice. Compared with controls, there were 30 and 20\% decreases in neuron number in the CD-deficient and combined Bax- and CD-deficient CA1 hippocampal region, respectively (Fig. 7A); there were 45 and $35 \%$ decreases in neuron number in CD-deficient and combined Bax- and CD-deficient cortex, respectively (Fig. $7 B$ ); and there were 30 and 25\% decreases in neuron number in CD-deficient and combined Bax- and CD-deficient subiculum, respectively (Fig. 7C). There were no statistical differences observed in neuron number between CD-deficient and combined Bax- and CD-deficient brain regions. Together, the findings presented in Figures 2-7 indicate that Bax deficiency prevents the induction of apoptosis by CD deficiency but does not prevent significant increases in neurodegeneration and neuron loss.

In addition to neurons with classical markers of apoptosis, neurons with a robust accumulation of autophagosomes have

A.

C.

E.
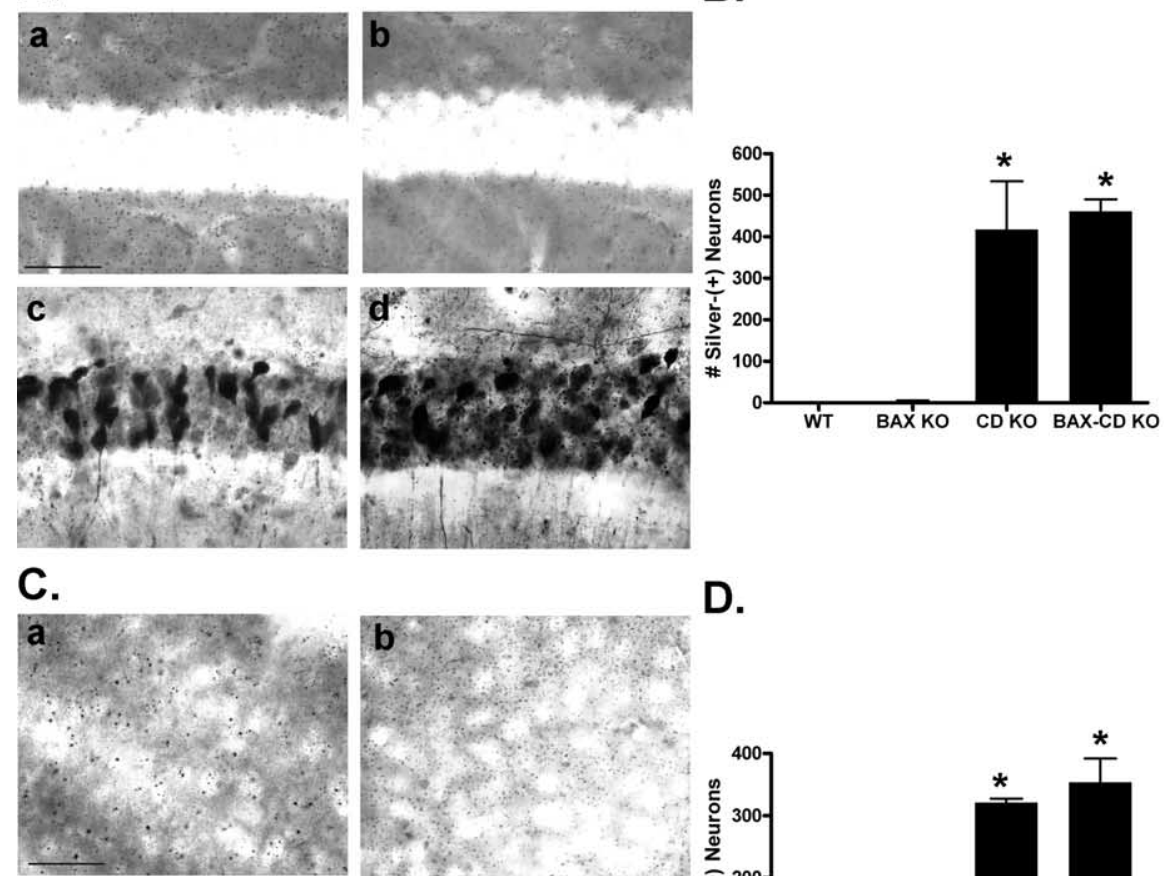

D.

B.
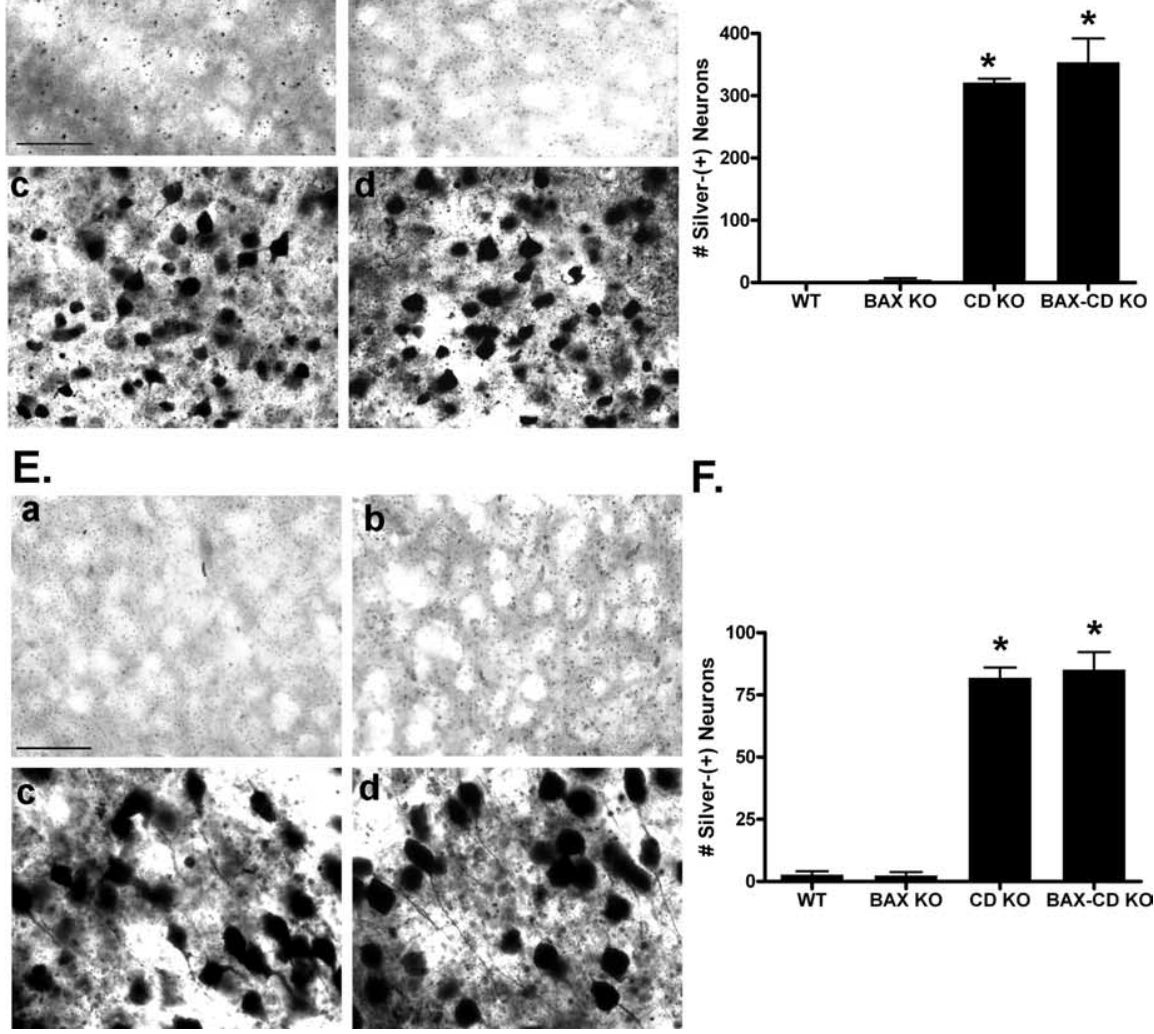

F.

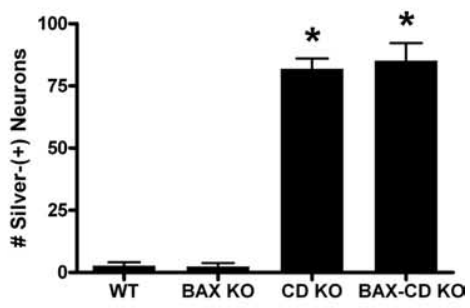

Figure 6. Bax deficiency does not prevent neurodegeneration induced by $C D$ deficiency. $A, C, E, D e O I m 0$ s silver stain analysis of the CA1 hippocampal region $(\boldsymbol{A})$, cortex $(\boldsymbol{C})$, and subiculum $(\boldsymbol{E})$ from P25-P26 WT control (a), Bax-deficient (b), CD-deficient (c), and combined Bax- and $(D$-deficient ( $\boldsymbol{d}$ ) mice. $\boldsymbol{B}, \boldsymbol{D}, \boldsymbol{F}$, The numbers of silver-positive, degenerating neurons were quantified for each brain region and for each genotype and are represented graphically as mean $\pm S E M .{ }^{*} p<0.05$, significantly different from WT and Bax-deficient mice. Results are representative of images obtained from three separate litters of mice. Scale bars, $50 \mu \mathrm{m}$. BAX KO, Bax-deficient; CD KO, CD-deficient; BAX-CD KO, combined Bax- and CD-deficient. also been identified in CD-deficient brain (Koike et al., 2000, 2005; Nakanishi et al., 2001; Walls et al., 2007), which defines a state of autophagic stress. To determine biochemically the potential contribution of autophagic stress to neurodegeneration induced by CD deficiency, levels of microtubule-associated protein LC3-II were assessed by Western blot (Fig. $8 A, B$ ). Under homeostatic conditions, LC3 is detected predominantly as a cytosolic 18 
A. Hippocampal CA1

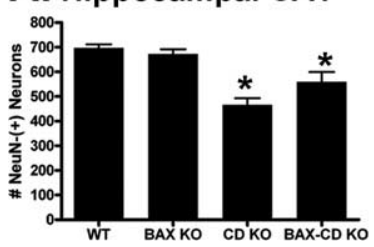

B. Cortex

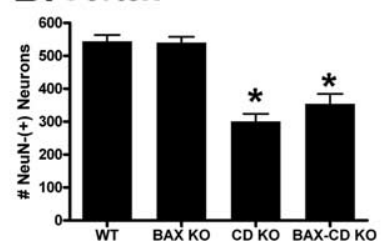

C. Subiculum

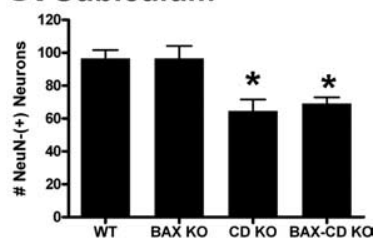

Figure 7. Bax deficiency does not prevent neuron loss induced by $C D$ deficiency. $A-C$, The numbers of NeuN-immunoreactive neurons were quantified in the CA1 hippocampal region $(\boldsymbol{A})$, cortex $(\boldsymbol{B})$, and subiculum $(\boldsymbol{C})$ from P25-P26 WT control, Baxdeficient (BAX KO), $C D$-deficient (CD KO), and combined Bax- and CD-deficient (BAX-CD KO) mice and are represented graphically as mean \pm SEM. ${ }^{*} p<0.05$, significantly different from WT and Bax-deficient mice. Results are representative of images obtained from at least seven separate litters of mice.

A.

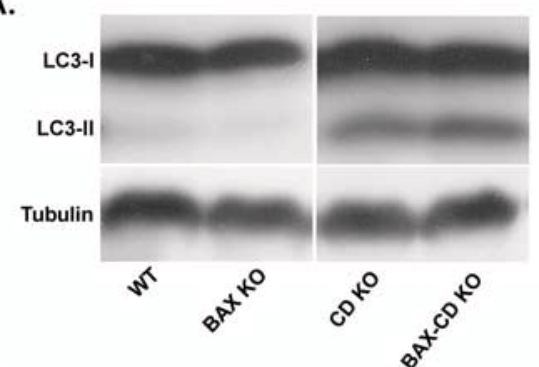

C.

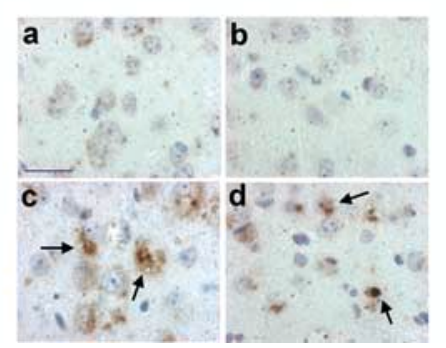

B.

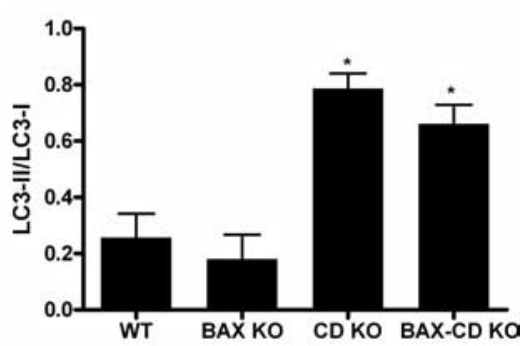

D.

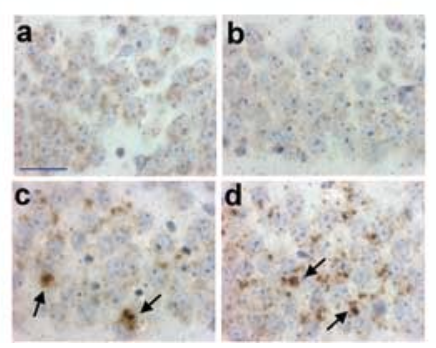

Figure 8. Bax deficiency does not prevent the CD deficiency-induced increase in LC3-II. A, Western blot analysis of whole-brain lysates obtained from P26 mice indicates the migration of LC3-I ( $18 \mathrm{kDa}$ ) and LC3-II (16 kDa). $\beta$-Tubulin, migrating at $\sim 50 \mathrm{kDa}$, was used as a loading control. $\boldsymbol{B}$, Results of Western blot for the ratio of LC3-II/LC3-I are represented graphically as mean \pm SEM. ${ }^{*} p<0.05$, significantly different from WT and Bax-deficient mice. $C, D$, Immunohistochemical analysis of $L(C 3$ in the cortex and CA1 hippocampal region from P26 WT control (a), Bax-deficient (b), CD-deficient (c), and combined Bax- and CD-deficient (d) mice. Arrows indicate cells with clumped, positive immunoreactivity for $L C 3$. Results are representative of measurements from at least three separate litters of animals. Scale bars, $20 \mu \mathrm{m}$. BAX KO, Bax-deficient; CD KO, CD-deficient; BAX-CD K0, combined Baxand CD-deficient.

$\mathrm{kDa}$ protein (LC3-I). However, under conditions of autophagic stress that exhibit an accumulation of autophagosomes, LC3 predominates as the post-translationally modified, $16 \mathrm{kDa}$ LC3-II, which is thought to associate exclusively with membranes of autophagosomes (Kabeya et al., 2000). Western blot analysis of lysates from WT and Bax-deficient brain indicated moderate levels of the cytosolic LC3-I and very low levels of LC3-II. Conversely, CD deficiency induced a dramatic and significant increase in LC3-II, an effect that was similar in combined Bax- and CDdeficient brains. LC3 levels and subcellular distribution were also evaluated by immunohistochemistry in the CA1 hippocampal region (Fig. $8 C$ ) and cortex (Fig. $8 D$ ). The intensity of LC3 staining was much more robust in the $\mathrm{CD}$-deficient and combined Bax- and CD-deficient CA1 hippocampal region and cortex compared with brains of WT and Bax-deficient control mice and appeared in large intracytoplasmic aggregates, consistent with the accumulation of autophagosomes. The antibody used to detect LC3 does not differentiate between LC3-I and LC3-II. However, the dramatic increase in LC3 immunoreactivity and its clumped, granular appearance in CD-deficient neurons corre- lated well with that observed for LC3-II by Western blot, which together indicate autophagic vacuole accumulation and resultant induction of autophagic stress in CD-deficient brains in the absence of Bax.

The disruption of lysosome function by CD deficiency is known to result in the accumulation of undigested, autofluorescent lipopigment in lysosomes and/or accumulated autophagosomes (Koike et al., 2000) and may be associated with the induction of autophagic stress in CDdeficient brains. Autofluorescent material (expressed as average autofluorescence units) in the cortex (Fig. 9A,B), subiculum (Fig. 9C,D), thalamus (Fig. 9E,F), and CA1-CA2 hippocampal region (Fig. $9 G, H)$ was quantified as a function of genotype. Minimal autofluorescence was detected in WT or Bax-deficient control brains. In contrast, $\mathrm{CD}$ deficiency induced a robust and significant increase in average autofluorescence units in each brain region analyzed, an effect that was statistically similar in combined Bax- and CDdeficient brains.

\section{Discussion}

CD-deficient mice exhibit accelerated neurodegeneration concurrent with morphological and biochemical features of apoptosis and autophagic stress and serve as a useful model for the study of neurodegeneration associated with lysosome dysfunction, in particular as it relates to NCL. The targeted deletion of bax, a critical mediator of the intrinsic apoptotic pathway, dramatically attenuates activation of caspase-3 in CD-deficient brain, thus allowing for the contribution of Baxdependent apoptosis in CD deficiencyinduced neurodegeneration to be determined. Our findings indicate persistent CD deficiency-induced neuropathology that occurs in the absence of Bax-dependent activation of caspase- 3 and apoptosis. This study not only suggests a limited role for Bax-dependent apoptosis in CD deficiency-induced neurodegeneration but also emphasizes the importance of caspaseindependent neuron death and neurodegeneration in models of lysosomal storage disorders.

Morphological and biochemical markers of apoptosis are observed in CD-deficient mouse brain (Figs. 2-5), which corroborates results of previous studies indicating activation of caspase- 9 and caspase- 3 in CD-deficient mouse neurons and increased TUNEL reactivity in large neurons of CD-deficient cortex and thalamus (Nakanishi et al., 2001; Koike et al., 2003). The targeted deletion of bax dramatically attenuates the activation of caspase- 3 (Fig. 5) that results from CD deficiency and is associated with a reduction in apoptotic-like morphology. However, Bax deficiency neither alters the severity of neurodegeneration (Fig. 6) nor attenuates neuron loss (Fig. 7) in any brain region assessed in this study (cortex, CA1-CA2 hippocampal region, or subiculum), which indicates that neuron death and neurodegeneration 
in CD-deficient mice are primarily Bax and caspase independent. Although it is still unclear what caspase-independent pathways are activated in CD-deficient brains, it is likely that these alternative death pathways occur either concomitantly with apoptosis and/or are exacerbated in the absence of apoptosis. Furthermore, the near complete attenuation of caspase- 3 activity in combined Bax- and CD-deficient brains precludes a major contribution of the extrinsic (caspase-8mediated) apoptotic pathway in CDdeficient neuropathology, because activation of caspase- 8 is an upstream activator of caspase-3 (Fernandes-Alnemri et al., 1996).

Additional evidence in this study supporting a role for Bax-independent induction of neuropathology in CD deficiency includes the persistence of swollen, lipofuscin-laden neurons, pyknotic nuclei, cells exhibiting hypereosinophilic cytoplasm, and TUNEL-reactive cells in the cortex of combined Bax- and CDdeficient mice (Figs. 2, 3). It is not clear from our results whether these swollen, lipofuscin-laden neurons are indeed actively degenerating or committed to death in the face of persistent lysosome dysfunction. Such "swollen" neurons in CDdeficient brain have been shown previously by EM analysis to be surrounded by activated microglia, suggesting an active process of phagocytosis that would certainly predict the onset of neurodegeneration (Nakanishi et al., 2001). In addition, the persistence of TUNEL-reactive neurons in the cortex in the absence of apoptosis may be an indication of necrotic cell death, as defined previously using the TUNEL method (van Lookeren et al., 1995). However, compared with the cortex, the CA1-CA2 region of the hippocampus appears to be more sensitive to Bax-dependent apoptosis, because bax deletion in this brain region partially but significantly attenuates the percentage of TUNEL-reactive neurons and reduces the prevalence of apoptotic morphology (Fig. 3 ). Nevertheless, markers of neurodegeneration, neuron loss, lysosome dysfunction, and autophagic stress all persist in combined Bax- and CD-deficient hippocampus, which suggests that Bax-independent neuron death and neurodegeneration still play a major role in this brain region.

Autophagic stress is evidenced as early as $\mathrm{P} 1$ in CD-deficient mouse brain by an increase in autophagosomes/autolysosomelike bodies within neurons, and the numbers of neurons exhibiting autophagic stress in CD-deficient mice are known to increase greatly with age (Koike et al., 2000, 2005). Small numbers of autophagosomes exist normally in cells under nonpathological conditions and serve to regulate the homeostatic turnover of cy-
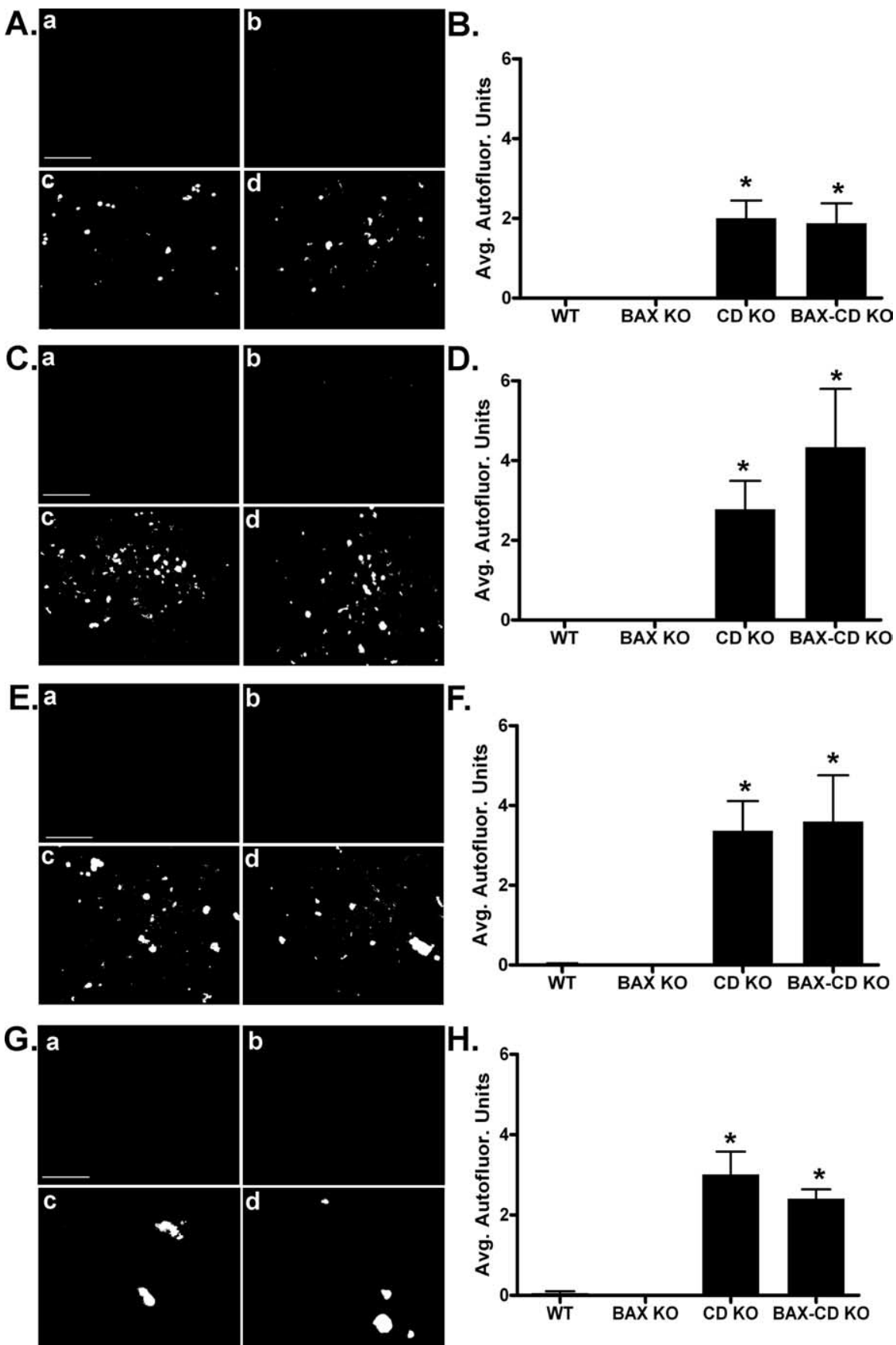

Figure 9. Bax deficiency does not prevent the accumulation of autofluorescent material induced by $C D$ deficiency. $A, C, E, G$, Representative images from the cortex $(\boldsymbol{A})$, subiculum $(\boldsymbol{C})$, thalamus $(\boldsymbol{E})$, and CA1-CA2 hippocampal region (G) from P25-P26 WT control (a), Bax-deficient (b), CD-deficient (c), and combined Bax-and CD-deficient (d) mice. $\boldsymbol{B}, \boldsymbol{D}, \boldsymbol{F}, \boldsymbol{H}$, Average autofluorescence (Avg. Autofluor.) units (see Materials and Methods for experimental details) for cortex $(\boldsymbol{B})$, subiculum $(\boldsymbol{D})$, thalamus $(\boldsymbol{F})$, and CA1-CA2 hippocampal region $(\boldsymbol{H})$ expressed as mean \pm SEM. ${ }^{*} p<0.05$, significantly different from WT and Bax-deficient mice. Each genotype is represented by at least four different litters of mice. Scale bars: $\boldsymbol{A}, \boldsymbol{C}, \boldsymbol{E}, 50 \mu \mathrm{m} ; \boldsymbol{G}, 20 \mu \mathrm{m}$. BAX K0, Bax-deficient; CD KO, CD-deficient; BAX-CD KO, combined Bax- and CD-deficient.
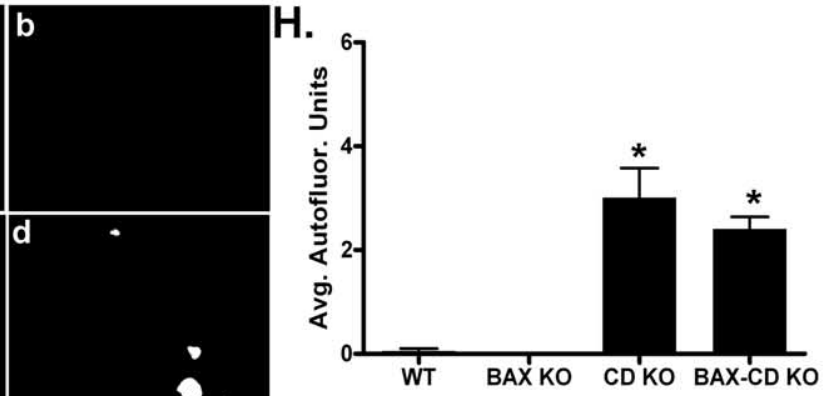

toplasm and organelles via shuttling materials to lysosomes for proteolytic degradation (Nixon and Cataldo, 1995). However, several recent studies have shown that metabolic stress or alterations in lysosomal function induce autophagic stress as defined by the dramatic cytoplasmic accumulation of autophagosomes, resulting from either the induction of autophagosome formation or from the inhibition of their degradation (Zaidi et al., 2001; Boya et al., 2003, 2005; Shimizu et al., 2004; Pattingre et al., 2005; Shacka et al., 2006a). With CD deficiency, lysosomes lack their 
principal aspartic acid protease, thus compromising an important means of proteolytic degradation (Saftig et al., 1995; Koike et al., 2000). Lysosomal dysfunction in CD-deficient brain most likely hinders the normal recycling of autophagosomes, thus precipitating their progressive and rapid accumulation in neurons. The effects of lysosomal dysfunction induced by CD deficiency are reflected in the present study not only by an increase in autophagosomes (Fig. 8) but also by the remarkable increase in neurons with swollen cytoplasm (Fig. 2) and autofluorescence (Fig. 9). Thus, although not directly assessed in this study, the manner in which autophagosomes accumulate in CD-deficient brain most likely results from an inhibition of autophagosome degradation rather from the stress-induced formation of nascent autophagosomes.

The accumulation of autophagosomes in CD-deficient brain is known to precede the appearance of apoptotic markers by several days (Koike et al., 2000, 2005; Nakanishi et al., 2001; Walls et al., 2007), suggesting that CD deficiency-induced lysosome dysfunction and resultant autophagic stress may contribute in part to the induction of Bax-dependent apoptosis. In the present study, markers of lysosome dysfunction, including swollen neurons laden with undigested storage material (Fig. 2) and autofluorescence (Fig. 9), along with markers of autophagic stress (LC3-II accumulation and the "clumped" appearance of LC3 by immunohistochemistry) (Fig. 8), are shown to persist in CDdeficient brain in the absence of Bax-dependent apoptosis. Previous examination of $\mathrm{CD}$-deficient cortex and thalamus revealed a lack of autophagic stress-induced morphology in TUNELreactive, apoptotic neurons (Nakanishi et al., 2001). Together, these results suggest that lysosome dysfunction and autophagic stress induced by CD deficiency may contribute to the induction of Bax-dependent apoptosis in addition to other forms of neuron death in CD-deficient brains. Although it is likely that lysosome dysfunction contributes to CD deficiency-induced neuron death, future studies are required to delineate a putative role of autophagic stress in mediating neuron death and neurodegeneration.

Many in vitro studies have shown that pharmacologically or chemically induced lysosome dysfunction causes an inhibition of autophagy and produces autophagic stress as evidenced by the rapid accumulation of autophagosomes, events that can trigger cell-autonomous, Bax-dependent apoptosis (Zaidi et al., 2001; Boya et al., 2005; Shacka et al., 2006a). Although the induction of Bax-dependent apoptosis may be related to lysosome dysfunction and the induction of autophagic stress in CD-deficient brain, results from the current study corroborates the hypothesis that apoptotic neurons can be distinct from those exhibiting autophagic stress (Nakanishi et al., 2001). Differences between the in vivo and in vitro effects of lysosomal dysfunction may be explained in part by the nature of the models used. In vitro studies use relatively pure cultures of dissociated cells/neurons with the stimulus and death occurring autonomously within several hours, whereas the in vivo model of CD deficiency is characterized by progressive neurodegeneration that is influenced potentially by non-cell-autonomous processes including microglial activation.

In CD-deficient brain, Bax-dependent apoptosis may result indirectly from microglial activation and phagocytosis of neurons exhibiting autophagic stress. CD-deficient neurons exhibiting an accumulation of autophagosomes have been shown to be surrounded by activated microglia, although it is not clear what the mechanisms are that mediate this response (Koike et al., 2000; Nakanishi et al., 2001). Activated microglia are known to express inducible nitric oxide synthase (iNOS), and increased iNOS has been documented in CD-deficient brain (Nakanishi et al., 2001). In addition, the systemic administration of NOS-selective inhibitors significantly attenuates the number of TUNEL-reactive neurons in CD-deficient brain without affecting those with autophagic stress-induced morphology (Nakanishi et al., 2001), which further emphasizes the contribution of multiple types of neuron death in CD-deficient brain. When produced in excess, NO may react with superoxide to form the potent oxidant peroxynitrite (Beckman et al., 1990; Ischiropoulos and Beckman, 2003). Both NO and peroxynitrite induce Bax-dependent apoptosis (Tamatani et al., 1998; Ghatan et al., 2000; Pervin et al., 2003; Shacka et al., 2006b) and may also contribute to Baxdependent apoptosis of CD-deficient neurons.

In light of recent reports indicating mutations in CD lead to a deficit in CD activity in NCL patients (Siintola et al., 2006; Steinfeld et al., 2006), it is tempting to speculate that the multiple types of neuron death observed in the CD-deficient mouse also exist in NCL, or at least in a subset of NCL patients, and that Baxdependent apoptosis also plays a limited role in NCL. In addition to NCL, lysosomal dysfunction and autophagic stress are currently implicated in many chronic neurodegenerative diseases of the aging brain, including Alzheimer's, Parkinson's, and Hungtinton's (Anglade et al., 1997; Roth, 2001; Bahr and Bendiske, 2002; Zhu et al., 2003; Ravikumar et al., 2004; Nixon et al., 2005). A potential role for Bax-independent neuron death and neurodegeneration in these diseases is particularly compelling in light of conflicting evidence that questions the importance of apoptosis in neurodegenerative disease pathogenesis (Jellinger and Stadelmann, 2000; Roth, 2001). Future studies of NCL and other neurodegenerative diseases should thus carefully consider the contribution of both apoptotic and autophagic stress-induced neuron death to their onset and progression. In addition, the development of agents that specifically target the downstream sequelae of lysosome dysfunction may prove useful in the treatment of NCL and other neurodegenerative diseases.

\section{References}

Anglade P, Vyas S, Javoy-Agid F, Herrero MT, Michel PP, Marquez J, MouattPrigent A, Ruberg M, Hirsch EC, Agid Y (1997) Apoptosis and autophagy in nigral neurons of patients with Parkinson's disease. Histol Histopathol 12:25-31.

Awano T, Katz ML, O’Brien DP, Taylor JF, Evans J, Khan S, Sohar I, Lobel P, Johnson GS (2006) A mutation in the cathepsin D gene (CTSD) in American Bulldogs with neuronal ceroid lipofuscinosis. Mol Genet Metab 87:341-348.

Bahr BA, Bendiske J (2002) The neuropathogenic contributions of lysosomal dysfunction. J Neurochem 83:481-489.

Beckman JS, Beckman TW, Chen J, Marshall PA, Freeman BA (1990) Apparent hydroxyl radical production by peroxynitrite: implications for endothelial injury from nitric oxide and superoxide. Proc Natl Acad Sci USA 87:1620-1624.

Boya P, Gonzalez-Polo RA, Poncet D, Andreau K, Vieira HL, Roumier T, Perfettini JL, Kroemer G (2003) Mitochondrial membrane permeabilization is a critical step of lysosome-initiated apoptosis induced by hydroxychloroquine. Oncogene 22:3927-3936.

Boya P, Gonzalez-Polo RA, Casares N, Perfettini JL, Dessen P, Larochette N, Metivier D, Meley D, Souquere S, Yoshimori T, Pierron G, Codogno P, Kroemer G (2005) Inhibition of macroautophagy triggers apoptosis. Mol Cell Biol 25:1025-1040.

Chu CT (2006) Autophagic stress in neuronal injury and disease. J Neuropathol Exp Neurol 65:423-432.

Deckwerth TL, Elliott JL, Knudson CM, Johnson Jr EM, Snider WD, Korsmeyer SJ (1996) BAX is required for neuronal death after trophic factor deprivation and during development. Neuron 17:401-411.

DeOlmos JS, Ingram WR (1971) An improved cupric-silver method for impregnation of axonal and terminal degeneration. Brain Res 33:523-529.

Fernandes-Alnemri T, Armstrong RC, Krebs J, Srinivasula SM, Wang L, Bull- 
rich F, Fritz LC, Trapani JA, Tomaselli KJ, Litwack G, Alnemri ES (1996) In vitro activation of CPP32 and Mch3 by Mch4, a novel human apoptotic cysteine protease containing two FADD-like domains. Proc Natl Acad Sci USA 93:7464-7469.

Ghatan S, Larner S, Kinoshita Y, Hetman M, Patel L, Xia Z, Youle RJ, Morrison RS (2000) p38 MAP kinase mediates bax translocation in nitric oxide-induced apoptosis in neurons. J Cell Biol 150:335-347.

Goebel HH, Wisniewski KE (2004) Current state of clinical and morphological features in human NCL. Brain Pathol 14:61-69.

Haltia M (2003) The neuronal ceroid-lipofuscinoses. J Neuropathol Exp Neurol 62:1-13.

Ischiropoulos H, Beckman JS (2003) Oxidative stress and nitration in neurodegeneration: cause, effect, or association? J Clin Invest 111:163-169.

Jellinger KA, Stadelmann CH (2000) The enigma of cell death in neurodegenerative disorders. J Neural Transm Suppl 121-36.

Jurgensmeier JM, Xie Z, Deveraux Q, Ellerby L, Bredesen D, Reed JC (1998) Bax directly induces release of cytochrome $\mathrm{c}$ from isolated mitochondria. Proc Natl Acad Sci USA 95:4997-5002.

Kabeya Y, Mizushima N, Ueno T, Yamamoto A, Kirisako T, Noda T, Kominami E, Ohsumi Y, Yoshimori T (2000) LC3, a mammalian homologue of yeast Apg8p, is localized in autophagosome membranes after processing. ЕMBO J 19:5720-5728.

Knudson CM, Tung KS, Tourtellotte WG, Brown GA, Korsmeyer SJ (1995) Bax-deficient mice with lymphoid hyperplasia and male germ cell death. Science 270:96-99.

Koike M, Nakanishi H, Saftig P, Ezaki J, Isahara K, Ohsawa Y, SchulzSchaeffer W, Watanabe T, Waguri S, Kametaka S, Shibata M, Yamamoto K, Kominami E, Peters C, von Figura K, Uchiyama Y (2000) Cathepsin $\mathrm{D}$ deficiency induces lysosomal storage with ceroid lipofuscin in mouse CNS neurons. J Neurosci 20:6898-6906.

Koike M, Shibata M, Ohsawa Y, Nakanishi H, Koga T, Kametaka S, Waguri S, Momoi T, Kominami E, Peters C, Figura K, Saftig P, Uchiyama Y (2003) Involvement of two different cell death pathways in retinal atrophy of cathepsin D-deficient mice. Mol Cell Neurosci 22:146-161.

Koike M, Shibata M, Waguri S, Yoshimura K, Tanida I, Kominami E, Gotow T, Peters C, von Figura K, Mizushima N, Saftig P, Uchiyama Y (2005) Participation of autophagy in storage of lysosomes in neurons from mouse models of neuronal ceroid-lipofuscinoses (Batten disease). Am J Pathol 167:1713-1728.

Lu Z, Dono K, Gotoh K, Shibata M, Koike M, Marubashi S, Miyamoto A, Takeda Y, Nagano H, Umeshita K, Uchiyama Y, Monden M (2005) Participation of autophagy in the degeneration process of rat hepatocytes after transplantation following prolonged cold preservation. Arch Histol Cytol 68:71-80.

Nakanishi H, Zhang J, Koike M, Nishioku T, Okamoto Y, Kominami E, von Figura K, Peters C, Yamamoto K, Saftig P, Uchiyama Y (2001) Involvement of nitric oxide released from microglia-macrophages in pathological changes of cathepsin D-deficient mice. J Neurosci 21:7526-7533.

Nixon RA, Cataldo AM (1995) The endosomal-lysosomal system of neurons: new roles. Trends Neurosci 18:489-496.

Nixon RA, Wegiel J, Kumar A, Yu WH, Peterhoff C, Cataldo A, Cuervo AM (2005) Extensive involvement of autophagy in Alzheimer disease: an immuno-electron microscopy study. J Neuropathol Exp Neurol 64:113-122.

Pattingre S, Tassa A, Qu X, Garuti R, Liang XH, Mizushima N, Packer M, Schneider MD, Levine B (2005) Bcl-2 antiapoptotic proteins inhibit Beclin 1-dependent autophagy. Cell 122:927-939.

Pervin S, Singh R, Chaudhuri G (2003) Nitric-oxide-induced Bax integration into the mitochondrial membrane commits MDA-MB-468 cells to apoptosis: essential role of Akt. Cancer Res 63:5470-5479.

Ravikumar B, Vacher C, Berger Z, Davies JE, Luo S, Oroz LG, Scaravilli F, Easton DF, Duden R, O'Kane CJ, Rubinsztein DC (2004) Inhibition of mTOR induces autophagy and reduces toxicity of polyglutamine expansions in fly and mouse models of Huntington disease. Nat Genet 36:585-595.
Reid WA, Valler MJ, Kay J (1986) Immunolocalization of cathepsin D in normal and neoplastic human tissues. J Clin Pathol 39:1323-1330.

Roth KA (2001) Caspases, apoptosis, and Alzheimer disease: causation, correlation, and confusion. J Neuropathol Exp Neurol 60:829-838.

Saftig P, Hetman M, Schmahl W, Weber K, Heine L, Mossmann H, Koster A, Hess B, Evers M, von Figura K, (1995) Mice deficient for the lysosomal proteinase cathepsin D exhibit progressive atrophy of the intestinal mucosa and profound destruction of lymphoid cells. EMBO J 14:3599-3608.

Shacka JJ, Roth KA (2005) Cathepsin deficiency as a model for neuronal ceroid lipofuscinoses. Am J Pathol 167:1473-1476.

Shacka JJ, Roth KA (2006) Bcl-2 family and the central nervous system: from rheostat to real complex. Cell Death Differ 13:1299-1304.

Shacka JJ, Klocke BJ, Shibata M, Uchiyama Y, Datta G, Schmidt RE, Roth KA (2006a) Bafilomycin Al inhibits chloroquine-induced death of cerebellar granule neurons. Mol Pharmacol 69:1125-1136.

Shacka JJ, Sahawneh MA, Gonzalez JD, Ye YZ, D’alessandro TL, Estevez AG (2006b) Two distinct signaling pathways regulate peroxynitrite-induced apoptosis in PC12 cells. Cell Death Differ 13:1506-1514.

Shimizu S, Kanaseki T, Mizushima N, Mizuta T, Arakawa-Kobayashi S, Thompson CB, Tsujimoto Y (2004) Role of Bcl-2 family proteins in a non-apoptotic programmed cell death dependent on autophagy genes. Nat Cell Biol 6:1221-1228.

Shindler KS, Latham CB, Roth KA (1997) Bax deficiency prevents the increased cell death of immature neurons in bcl-x-deficient mice. J Neurosci 17:3112-3119.

Shindler KS, Yunker AM, Cahn R, Zha J, Korsmeyer SJ, Roth KA (1998) Trophic support promotes survival of bcl-x-deficient telencephalic cells in vitro. Cell Death Differ 5:901-910.

Siintola E, Partanen S, Stromme P, Haapanen A, Haltia M, Maehlen J, Lehesjoki AE, Tyynela J (2006) Cathepsin D deficiency underlies congenital human neuronal ceroid-lipofuscinosis. Brain 129:1438-1445.

Steinfeld R, Reinhardt K, Schreiber K, Hillebrand M, Kraetzner R, Bruck W, Saftig P, Gartner J (2006) Cathepsin D deficiency is associated with a human neurodegenerative disorder. Am J Hum Genet 78:988-998.

Tamatani M, Ogawa S, Nunez G, Tohyama M (1998) Growth factors prevent changes in Bcl-2 and Bax expression and neuronal apoptosis induced by nitric oxide. Cell Death Differ 5:911-919.

Tornusciolo DR, Schmidt RE, Roth KA (1995) Simultaneous detection of TDT-mediated dUTP-biotin nick end-labeling (TUNEL)-positive cells and multiple immunohistochemical markers in single tissue sections. Biotechniques 19:800-805.

Tyynela J, Sohar I, Sleat DE, Gin RM, Donnelly RJ, Baumann M, Haltia M, Lobel P (2000) A mutation in the ovine cathepsin D gene causes a congenital lysosomal storage disease with profound neurodegeneration. EMBO J 19:2786-2792.

van Lookeren CM, Lucassen PJ, Vermeulen JP, Balazs R (1995) NMDA and kainate induce internucleosomal DNA cleavage associated with both apoptotic and necrotic cell death in the neonatal rat brain. Eur J Neurosci 7:1627-1640.

Walls KC, Klocke BJ, Saftig P, Shibata M, Uchiyama Y, Roth KA, Shacka JJ (2007) Altered regulation of phosphatidylinositol 3-kinase signaling in cathepsin D-deficient brain. Autophagy, in press.

Whitaker JN, Rhodes RH (1983) The distribution of cathepsin D in rat tissues determined by immunocytochemistry. Am J Anat 166:417-428.

White FA, Keller-Peck CR, Knudson CM, Korsmeyer SJ, Snider WD (1998) Widespread elimination of naturally occurring neuronal death in Baxdeficient mice. J Neurosci 18:1428-1439.

Zaidi AU, McDonough JS, Klocke BJ, Latham CB, Korsmeyer SJ, Flavell RA, Schmidt RE, Roth KA (2001) Chloroquine-induced neuronal cell death is p53 and $\mathrm{Bcl}-2$ family-dependent but caspase-independent. J Neuropathol Exp Neurol 60:937-945.

Zhu JH, Guo F, Shelburne J, Watkins S, Chu CT (2003) Localization of phosphorylated ERK/MAP kinases to mitochondria and autophagosomes in Lewy body diseases. Brain Pathol 13:473-481. 